\title{
Nanoparticulate Radiolabelled Quinolines Detect Amyloid Plaques in Mouse Models of Alzheimer's Disease
}

\author{
Celeste A. Roney, ${ }^{1}$ Veera Arora, ${ }^{1}$ Padmakar V. Kulkarni, ${ }^{1,2}$ Peter P. Antich, ${ }^{1,2}$ \\ and Frederick J. Bonte ${ }^{1,2}$ \\ ${ }^{1}$ Division of Radiological Sciences, Department of Radiology, University of Texas Southwestern Medical Center at Dallas,
Dallas, TX 75390, USA
${ }^{2}$ The Nuclear Medicine Center, University of Texas Southwestern Medical Center at Dallas, Dallas, TX 75390, USA
}

Correspondence should be addressed to Frederick J. Bonte, frederick.bonte@utsouthwestern.edu

Received 17 September 2009; Accepted 19 December 2009

Recommended by Craig Atwood

Detecting aggregated amyloid peptides (A $\beta$ plaques) presents targets for developing biomarkers of Alzheimer's disease (AD). Polymeric n-butyl-2-cyanoacrylate (PBCA) nanoparticles (NPs) were encapsulated with radiolabelled amyloid affinity ${ }^{125} \mathrm{I}-$ clioquinol (CQ, 5-chloro-7-iodo-8-hydroxyquinoline) as in vivo probes. ${ }^{125} \mathrm{I}$-CQ-PBCA NPs crossed the BBB $(2.3 \pm 0.9 \mathrm{ID} / \mathrm{g})(P<$ $.05)$ in the WT mouse $(N=210)$, compared to ${ }^{125} \mathrm{I}-\mathrm{CQ}(1.0 \pm 0.4 \mathrm{ID} / \mathrm{g}) .{ }^{125} \mathrm{I}-\mathrm{CQ}-\mathrm{PBCA} \mathrm{NP}$ brain uptake increased in AD transgenic mice (APP/PS1) versus WT $\left(N=38 ; 2.54 \times 10^{5} \pm 5.31 \times 10^{4} \mathrm{DLU} / \mathrm{mm}^{2}\right.$; versus $\left.1.98 \times 10^{5} \pm 2.22 \times 10^{4} \mathrm{DLU} / \mathrm{mm}^{2}\right)$ and in APP/PS1/Tau. Brain increases were in mice intracranially injected with aggregated $\mathrm{A} \beta_{42}$ peptide $\left(N=17 ; 7.19 \times 10^{5} \pm 1.25 \times 10^{5} \mathrm{DLU} / \mathrm{mm}^{2}\right)$, versus WT $\left(6.07 \times 10^{5} \pm 7.47 \times 10^{4} \mathrm{DLU} / \mathrm{mm}^{2}\right)$. Storage phosphor imaging and histopathological staining of the plaques, $\mathrm{Fe}^{2+}$ and $\mathrm{Cu}^{2+}$, validated results. ${ }^{125} \mathrm{I}-\mathrm{CQ}$-PBCA NPs have specificity for A $\beta$ in vitro and in vivo and are promising as in vivo SPECT $\left({ }^{123} \mathrm{I}\right)$, or PET $\left({ }^{124} \mathrm{I}\right)$ amyloid imaging agents.

Copyright ( $) 2009$ Celeste A. Roney et al. This is an open access article distributed under the Creative Commons Attribution License, which permits unrestricted use, distribution, and reproduction in any medium, provided the original work is properly cited.

\section{Introduction}

Alzheimer's disease (AD), a neurodegenerative disorder principally of the elderly, is the most prevalent form of dementia. The cognitive decline associated with AD drastically affects the social and behavioral skills of patients living with this disease. Notwithstanding the social impact, AD, also places great financial burdens on patients, families, and the community as a whole. Furthermore, therapeutic strategies to probe the central nervous system (CNS) are limited by the restrictive tight junctions at the endothelial cells of the blood brain barrier (BBB). To overcome the impositions of the $\mathrm{BBB}$, polymeric biocompatible drug carriers have been applied to the CNS for many applications, including cancers; however, the field of drug carrier technology is not well developed in AD research [1]. Polymeric nanoparticles are promising candidates in the investigation of $\mathrm{AD}$ since they are capable of opening tight junctions [2] crossing the BBB [3], high drug loading capacities, and targeting the mutant proteins of Alzheimer's [4,5].
Markedly elevated concentrations of zinc, copper, and iron in $\mathrm{A} \beta$ deposits of the $\mathrm{AD}$ brain are well documented in the literature $[6,7]$. Cherny et al. have employed the antibiotic and $\mathrm{Cu} / \mathrm{Zn}$-selective metal chelator, $\mathrm{CQ}$, to inhibit $\mathrm{A} \beta$ accumulations in AD APP2576 transgenic (Tg) mice. Oral treatments with $\mathrm{CQ}$ reduced $\mathrm{A} \beta$ deposition by $\approx 49 \%$ and produced no neurotoxicity in a blinded study. The authors [8] speculate that CQ's action on the peptide may facilitate $\mathrm{H}_{2} \mathrm{O}_{2}$ inhibition [8]. Moreover, the authors validated the selectivity of CQ since systemic metal depletion was not found [8]; CQ does not deplete brain tissue of metals, but rather binds to the $\mathrm{A} \beta$-metal complex itself.

In in vivo experiments with nontransgenic animals treated with CQ, Yassin et al. confirmed significant decreases in the cerebral concentrations of $\mathrm{Cu}, \mathrm{Zn}$, and Fe metal ions [9]. However, in Cherny's studies of APP2576 Tg mice, the inhibition of $\mathrm{A} \beta$ deposition by $\mathrm{CQ}$ caused significant increases in the cerebral concentrations of $\mathrm{Cu}$ and $\mathrm{Zn}$ [8]. This is because APP transgene expression reduces $\mathrm{Cu}$ and $\mathrm{Zn}$ 
levels in vivo, while the $\mathrm{A} \beta$ concentration steadily rises [10]. There are a few possibilities for the decrease. For example, the metal ions may be effluxed by $\mathrm{A} \beta$, or $\mathrm{APP} / \mathrm{A} \beta$ may prevent reuptake of the ions [11]. Despite the mechanism, CQ prevents uptake; of the metals by the protein. This action affords metallic access to peripheral brain tissue, and the metal ion concentration increases. Since AD is a syndrome of metal dyshomeostasis, CQ may be able to restore the metal metabolism to its normal state.

Opazo et al. [12] have explored the $\mathrm{A} \beta$-plaque binding properties of radioiodinated CQ in APP2576 Tg mice ([125 I]CQ; higher brain retention compared with controls on autoradiography), and in $\mathrm{AD}$ patients ([$\left.{ }^{123} \mathrm{I}\right] \mathrm{CQ}$; more rapid uptake compared with age-matched healthy controls). SPECT imaging was limited due to low tracer uptake, however, $\mathrm{CQ}$ was shown to localize with $\mathrm{A} \beta$ pathology [12]. Notably, the authors [12] synthetically precipitated $A \beta_{1-40}$ and $A \beta_{1-42}$ by in vitro methods. The synthetic $\beta$-amyloid demonstrated ${ }^{125} \mathrm{ICQ}$ saturation, which was directed by $\mathrm{Zn}^{2+}$; the ${ }^{125}$ ICQ binding was displaced by the heavy metals $\mathrm{Zn}^{2+}$ and $\mathrm{Cu}^{2+}$, the metal chelator DTPA, and the amyloid affinity dye Congo red. Enhancement of $\mathrm{Zn}^{2+}{ }^{125} \mathrm{ICQ}$ was also shown in concentrated fractions of $A \beta$ from postmortem $\mathrm{AD}$ brain. These results validate the effectiveness of CQ as a $\beta$-amyloid detection agent, and most importantly, correlate literature evidence linking $\mathrm{A} \beta$ deposition in $\mathrm{AD}$ and cerebral heavy metals $[5,8]$.

Our preliminary studies with ${ }^{125} \mathrm{ICQ}$ showed that the agent crossed the $\mathrm{BBB}$, but was retained too briefly for effective chelation. Therefore, a drug carrier is required to improve the extravascular retention of ${ }^{125} \mathrm{ICQ}$. Polymeric butylcyanoacrylate (PBCA) nanoparticles (NPs) were chosen as the drug carrier. Here, we report that ${ }^{125} \mathrm{I}$-CQ-BCA NPs act as targeted drug carriers with an affinity for amyloid plaques. ${ }^{125} \mathrm{I}$-CQ-PBCA NPs are promising candidates for in vivo brain imaging of the amyloid plaques.

\section{Methods}

2.1. Radioiodination of Clioquinol. CQ was radiolabelled with ${ }^{125} \mathrm{I}$ (Perkin Elmer, Waltham, MA) by the Chloramine$\mathrm{T}$ (CT) method of radioiodination. Purification of ${ }^{125} \mathrm{I}-\mathrm{CQ}$ was performed by solvent extraction in dichloromethane (DCM) and $\mathrm{H}_{2} \mathrm{O}$. The organic layer (e.g., DCM) was evaporated under $\mathrm{N}_{2}$ gas, and the ${ }^{125} \mathrm{I}-\mathrm{CQ}$ was dissolved in dimethylsulfoxide (DMSO, $1 \mathrm{~mL}$ ). The purification of ${ }^{125}$ I-CQ was analyzed by thin layer radiochromatography (RTLC). The specific purity was $>95 \%$.

2.2. Synthesis of Polymeric Nanoparticles. The NPs were polymerized as per the modified procedure of Kreuter et al. [13]. A polymerization medium was prepared containing Dextran 70000 and Tween-80 (polysorbate 80) (both at a concentration of $1 \%$ each in $0.1 \mathrm{~N} \mathrm{HCl}$ ) (Sigma, USA); $5.05 \times 10^{6} \mathrm{~Bq}^{125} \mathrm{I}$-CQ was added to the solution just prior to the addition of butylcyanoacrylate (BCA) monomer. A $1 \%$ $(\mathrm{w} / \mathrm{v})$ butylcyanoacrylate solution (Sichelwerke, Hannover, Germany) was added drop wise during constant magnetic stirring at $400 \mathrm{rpm}$. After 3 hours of polymerization, the NP suspension was neutralized with $0.1 \mathrm{~N} \mathrm{NaOH}$ to complete the polymerization. This solution was filtered with a $0.2 \mu \mathrm{m}$ filter and purified by ultracentrifugation (Beckman Coulter, Fullerton, CA; $45 \mathrm{~K} \mathrm{rpm}, 1 \mathrm{~h}$ ). The pellet was washed and redispersed in water, which contained $1 \%$ Tween-80. The PBCA nanoparticles were then overcoated with $1 \%$ Tween- 80 by stirring for 30 minutes in phosphate buffer solution (1X PBS), just before in vivo administration. Nanoparticle size $(45 \mathrm{~nm})$ was determined by a Zetasizer 3000 HS (Malvern, Worcestershire, UK).

2.3. In Vitro Labeling of Amyloid Plaques. Cortical frontal $\mathrm{AD}$ and control brain tissue were obtained from the tissue bank, as approved by our Institutional Review Board $(800 \mu \mathrm{L}$ buffer solution of $0.1 \%$ FBS) in the presence of ${ }^{125} \mathrm{I}-\mathrm{CQ}$ $\left(1.17 \times 10^{4} \mathrm{~Bq}\right.$ in $100 \mu \mathrm{L}$ PBS $)$. Brain homogenates were microcentrifuged (13 KRPM, 15 minutes) and the percent binding was calculated. The experimental results provided evidence of preferential binding by ${ }^{125} \mathrm{I}-\mathrm{CQ}$ to the $\mathrm{AD}$ brain tissue, as compared to cortical control brain tissue.

2.4. Aggregation of the $A \beta_{42}$ Peptide. Amyloid protein (142), $A \beta_{42},(0.5 \mathrm{mg})$ was purchased from Bachem California (Torrance, CA), and dissolved in 1.15 mL PBS (pH 7.4) to a final concentration of $435 \mu \mathrm{g} / \mathrm{mL}(100 \mu \mathrm{M})$ by magnetically stirring the solution in a closed vessel at 1200 RPM for 7 days at room temperature [14]. After 7 days, the aggregated peptide suspension was visibly cloudy. The aggregated $A \beta_{42}$ was stored in $100 \mu \mathrm{L}$ aliquots at $-20^{\circ} \mathrm{C}$ until use.

2.5. Intrahippocampal Sterotaxic Injection of Aggregated $A \beta_{42}$. All use of animals was in compliance with the regulations of the Animal Resources Center (ARC) of UT Southwestern Medical Center, and approved by the Institutional Review Board (IRB). All mice were anesthetized intraperitoneal (IP) with $100-150 \mu \mathrm{L}$ of Ketamine HCL (Sigma, St. Louis, Mo). Wild type BALB/C mice (Charles River Laboratories, Wilmington, MA; $N=17$ ) were injected by direct stereotaxis (Model 900 Small Animal Stereotaxic Instrument, David Kopf Instruments, Tujunga, CA) with the aggregated $\mathrm{A} \beta_{42}$ peptide at a concentration of $1 \mu \mathrm{g} / 1 \mu \mathrm{L}$, and at a constant flow rate of 60 seconds. A lubricant was placed into the eyes of the mice to prevent over-drying during the experiments. The mice received unilateral injections of either saline or the $\mathrm{A} \beta$ peptide aggregate. The injection location corresponded to the mouse brain hippocampus structure $\mathrm{CA} 1$, at coordinates $-1.5,-1.0$, and -1.8 , relative to Bregma $[15,16]$. The peptide was allowed to grow for 7 days, during and after which time cognitive tests for behavior (Y-maze) were performed.

2.6. In Vivo Imaging of the Plaques by Nanoparticle Administration. The ${ }^{125} \mathrm{I}-\mathrm{CQ}-\mathrm{BCA}$ NPs $(1-3 \mathrm{mg})$ were administered to $\mathrm{AD}$ mouse transgenic models by lateral tail vein injection. The $A \beta_{42}$ mice received ${ }^{125}$ I-CQ-BCA NPs at 8 days postinjection of the peptide. 
In vivo Storage Phosphor autoradiography (Perkin Elmer Cyclone Storage Phosphor Imaging System; OptiQuant Imaging Software) was used to determine the relative qualitative differences in the brain uptakes of ${ }^{125} \mathrm{I}-\mathrm{CQ}$ by the AD mouse models and wildtype control mice. Briefly, each mouse was anesthetized I.P. with $100-150 \mu \mathrm{L}$ of Ketamine $\mathrm{HCl}$ (Sigma Aldrich, USA) throughout the duration (5-90 minutes) of the imaging experiment. Postinjection of the radiotracer, the mouse was placed on the phosphor screen with a lead sheet between the film and the animal's body in such a way that the only exposed body part was the head. In this way, background radiation from the body was minimized, and the activity source projected onto the film was from the animal's head region only. Regions of interest (ROI) were drawn in the brain space to obtain information in semiquantitative units (Dynamic Light Units, DLU) per volume space $\left(\mathrm{DLU} / \mathrm{mm}^{2}\right)$.

At the conclusion of the imaging experiments, the mice were sacrificed by cardiac perfusion through the left ventricle with $4 \%$ paraformaldehyde. The brains were harvested and stored in formalin, before being embedded in wax, and sectioned at a $5 \mu \mathrm{m}$ slice thickness. The slices were stained with Congo red (amyloid plaques), Prussian blue $\left(\mathrm{Fe}^{2+}\right)$, and Rubeanic acid $\left(\mathrm{Cu}^{2+}\right)$.

2.7. AD Transgenic Mice. Mice with a double mutation (APP/PS1) for Alzheimer's disease were commercially purchased $(N=5)$ from The Jackson Laboratories (Bar Harbor, ME; strain B6C3-Tg(APPswe, PSEN1dE9)85Dbo/J). This particular mouse model corresponds to a form of early onset disease and expresses a mutant human presenilin 1 and a chimeric mouse/human amyloid precursor protein $\left(\mathrm{APP}_{\text {Swe }}\right)$. The expression of both transgenes was directed by the mouse prion protein promoter. The APPswePS1 strain was developed on a B6C3HF2 background. The chimeric APP was modified to encode the Sweedish mutations K595N/M596L in order to elevate the amount ot $\mathrm{A} \beta$ produced from the transgene, by favoring processing through the beta-secretase pathway. Mice with the double mutation (APP/PS1) were generously donated $(N=33)$ by Dr. David Russell (UT Southwestern). Mice with the triple mutation (APP/PS1/Tau) were generated $(N=2)$ by Dr. Malu Tansey (UT Southwestern), to express the knock-in human presenilin 1 mutation, mutant Tau, and the Swedish APP mutation.

\section{Statistical Analysis}

Data were entered into Excel worksheets (Microsoft Corporation, Redmond, WA), and analyzed using the non-paired, two-tailed Student's $t$-test with unequal variance. $P<.05$ was regarded as significant.

\section{Results}

4.1. In Vitro Binding of ${ }^{125} \mathrm{I}-\mathrm{CQ}$ to Brain Tissue. ${ }^{125} \mathrm{ICQ}$ was used in in vitro assays of human postmortem frontal cortex to test the affinity of the radiolabelled chelator for amyloid

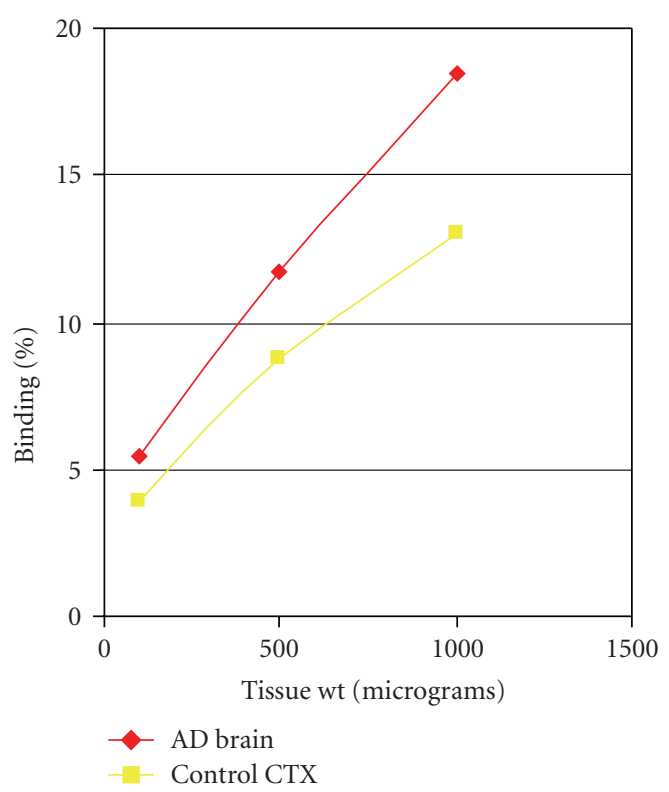

FIGURE 1: In vitro binding of ${ }^{125} \mathrm{I}-\mathrm{CQ}$ to brain homogenates.

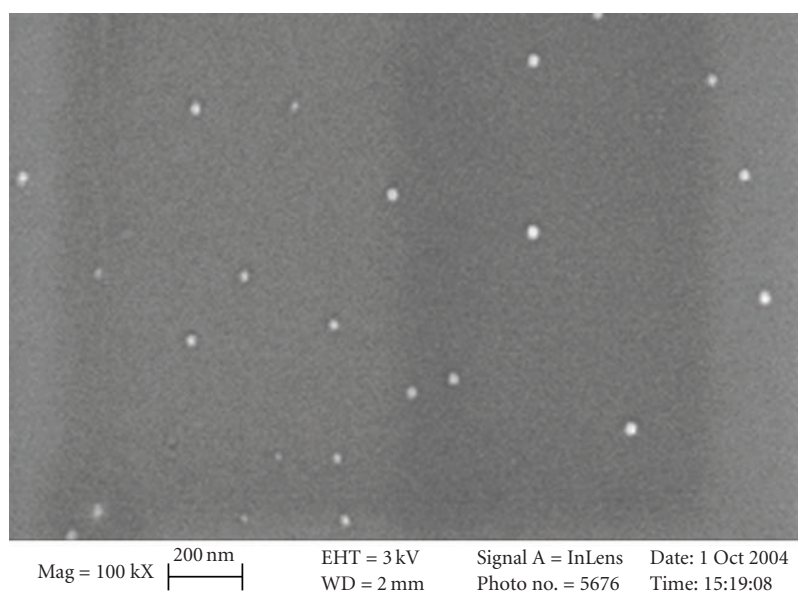

FIgURE 2: PBCA Nanoparticles.

plaques. Age-matched normal specimens were tested as control samples; no AD pathology was found in normal tissue (i.e., plaques and tangles in the brain). Experimental results (Figure 1) provide evidence of preferential binding by ${ }^{125} \mathrm{I}-\mathrm{CQ}$ to the AD brain tissue ( $1000 \mu \mathrm{g}, 18.5 \%$ binding), as compared to cortical control brain tissue $(1000 \mu \mathrm{g}$, $13 \%$ binding). Therefore, an amyloid-affinity drug could be successfully radiolabelled; the radioligand discriminated between $\mathrm{AD}$ brain tissue and control brain tissue.

4.2. In Vivo Biodistribution of ${ }^{125} I-C Q$ BCA NPs. The BCA monomer was polymerized with small particulate diameter $(45 \mathrm{~nm})$ and with uniform size distribution (Figure 2). In vivo biodistribution experiments showed that the free ${ }^{125} \mathrm{I}$ CQ had a rapid brain uptake, as well as rapid blood clearance in normal mice (Table 1). Furthermore, ${ }^{125} \mathrm{I}-\mathrm{CQ}$ cleared the brain quickly; the $\% \mathrm{ID} / \mathrm{g}$ for the brain at 2 minutes was 
TABLE 1: In vivo biodistribution of ${ }^{125} \mathrm{I}-\mathrm{CQ}$ by IV administration in wild type mice $(n=105)$ (table represents organ uptakes in $\%$ ID/g \pm $\mathrm{SD})$.

\begin{tabular}{lccccc}
\hline Time $(\mathrm{min})$ & Brain & Blood & Spleen & Liver & Brain : blood ratio \\
\hline 2 & $1 \pm 0.4$ & $9.2 \pm 2.8$ & $2.6 \pm 0.1$ & $12.5 \pm 0.8$ & $0.1 \pm 0.1$ \\
5 & $1 \pm 0.3$ & $5.7 \pm 1.3$ & N/A & $15.5 \pm 5.6$ & $0.2 \pm 0.01$ \\
15 & $0.3 \pm 0.1$ & $2.9 \pm 0.7$ & $1.2 \pm 0.1$ & $3.8 \pm 0.7$ & $0.1 \pm 0.1$ \\
30 & $0.3 \pm 0.1$ & $2.3 \pm 0.4$ & $1.3 \pm 1$ & $5.2 \pm 2.2$ & $0.1 \pm 0.1$ \\
60 & $0.1 \pm 0.1$ & $1.3 \pm 0.7$ & $0.5 \pm 0.3$ & $3.6 \pm 2.3$ & $0.3 \pm 0.5$ \\
120 & $0.03 \pm 0.03$ & $0.1 \pm 0.1$ & $0.6 \pm 0.4$ & $1.3 \pm 0.5$ & $0.2 \pm 0.1$ \\
240 & $0.04 \pm 0.02$ & $0.6 \pm 0.2$ & $0.4 \pm 0.3$ & $0.9 \pm 0.2$ & $0.1 \pm 0.1$ \\
\hline
\end{tabular}

$P=.05$

TABLE 2: In vivo biodistribution of ${ }^{125}$ I-CQ-BCA-NPs by IV administration in wild type mice $(n=105)$ (NP polymerized in the presence of $1 \%$ Dextran 70000 and 1\% Tween-80; the table represents organ uptakes in \%ID/g \pm SD).

\begin{tabular}{lccccc}
\hline Time $(\mathrm{min})$ & Brain & Blood & Spleen & Liver & Brain:bloodratio \\
\hline 2 & $2.3 \pm 0.9$ & $12 \pm 2.5$ & $1.6 \pm 0.4$ & $15 \pm 2.1$ & $0.2 \pm 0.1$ \\
5 & $1.5 \pm 0.9$ & $7.9 \pm 1.6$ & $1.8 \pm 0.5$ & $11.4 \pm 1.5$ & $0.2 \pm 0.1$ \\
15 & $0.5 \pm 0.2$ & $4.9 \pm 1.2$ & $1.4 \pm 0.5$ & $8.1 \pm 2.1$ & $0.1 \pm 0.1$ \\
30 & $0.3 \pm 0.1$ & $3.4 \pm 1.5$ & $0.9 \pm 0.7$ & $6.6 \pm 2.4$ & $0.1 \pm 0.0$ \\
60 & $0.2 \pm 0.1$ & $2.1 \pm 1.1$ & $0.5 \pm 0.3$ & $3.9 \pm 1.6$ & $0.1 \pm 0.0$ \\
120 & $0.03 \pm 0.0$ & $0.7 \pm 0.2$ & $0.2 \pm 0.03$ & $2.3 \pm 0.4$ & $0.1 \pm 0.0$ \\
240 & $0.02 \pm 0.0$ & $0.4 \pm 0.0$ & $0.1 \pm 0.1$ & $1.4 \pm 0.1$ & $0.01 \pm 0.0$ \\
\hline
\end{tabular}

$P=.05$

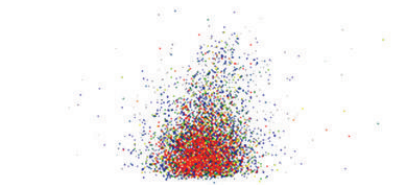

AD transgenic mouse with $7.56 \times 10^{5} \mathrm{CPM}^{125}$ ICQ BCA NPs

FIGURE 3: AD transgenic mice injected with ${ }^{125}$ ICQ BCA NPs and ${ }^{125} \mathrm{ICQ}$ (Mice aged $7 \mathrm{mo} ; 5$ minutes post injection).

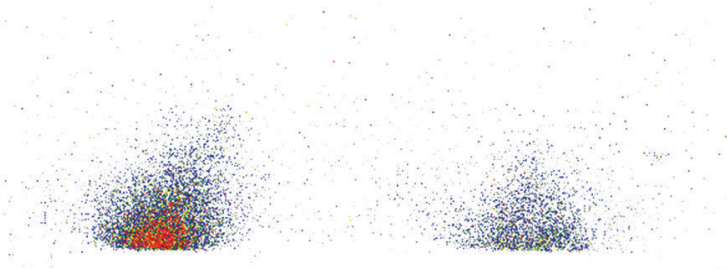

FIgure 4: AD transgenic mice injected with ${ }^{125}$ ICQ BCA NPs and ${ }^{125}$ ICQ (Mice aged 7 mo; 90 minutes post injection).

$0.99 \pm 0.40 \%$, and at 4 hours, it was $0.04 \pm 0.02 \%$. Table 2 shows that when the ${ }^{125} \mathrm{I}-\mathrm{CQ}$ was encapsulated within the BCA NPs, the brain uptake was enhanced. At two minutes, the wild type mice exhibited $2.31 \pm 0.89 \%$ uptake of the ${ }^{125}$ I-CQ BCA NPs in the brain (Table 1). Brain and blood clearances of the ${ }^{125} \mathrm{I}-\mathrm{CQ}$ BCA NPs were rapid; the \% ID/g (brain) at 4 hours was $0.02 \%$. Together, Tables 1 and 2 show
TABLE 3: 7 month AD transgenic with 9 month control with ${ }^{125} \mathrm{ICQ}$ BCA NPs in DLU/mm² $($ mean $\pm \mathrm{SD}) ;(n=4)$.

\begin{tabular}{lcc}
\hline Time (mins) & 9 mo control & 7 mo AD transgenic \\
\hline 10 & $1.64 \times 10^{5} \pm 1.75 \times 10^{4}$ & $2.26 \times 10^{5} \pm 4.22 \times 10^{5}$ \\
30 & $1.76 \times 10^{5} \pm 3.49 \times 10^{4}$ & $1.63 \times 10^{5} \pm 2.44 \times 10^{4}$ \\
60 & $1.06 \times 10^{5} \pm 1.57 \times 10^{4}$ & $7.04 \times 10^{4} \pm 1.14 \times 10^{4}$ \\
90 & $1.98 \times 10^{5} \pm 2.22 \times 10^{4}$ & $2.54 \times 10^{5} \pm 5.31 \times 10^{4}$ \\
\hline$P=.05 ; 1$ mg BCA NP; 795,338 CPM ${ }^{125}$ ICQ.
\end{tabular}

that ${ }^{125} \mathrm{I}-\mathrm{CQ}$ BCA NPs have an increased brain uptake versus ${ }^{125} \mathrm{I}-\mathrm{CQ}$ in the wild type control mouse.

Table 3 shows similar results, in Dynamic Light Units $/ \mathrm{mm}^{2} \quad\left(\mathrm{DLU} / \mathrm{mm}^{2}\right)$, the unit described in autoradiographic imaging. For example, in the wildtype control mouse, at 10 minutes post injection the brain uptake of encapsulated ${ }^{125} \mathrm{I}-\mathrm{CQ}$ is $1.64 \times 10^{5} \pm 1.75 \times 10^{4}$ versus $2.26 \times 10^{5} \pm 4.22 \times 10^{5}$ in the $\mathrm{AD}$ transgenic mouse. Moreover, the brain uptake is significantly greater in the $\mathrm{AD}$ mouse at 90 minutes post injection of ${ }^{125} \mathrm{I}-\mathrm{CQ}$ BCA NPs $\left(1.98 \times 10^{5} \pm 2.22 \times 10^{4}\right.$ wildtype versus $2.54 \times 10^{5} \pm 5.31 \times 10^{4}$ AD transgenic)

Triple transgenic mice, for which mutations in the APP and Tau genes result in amyloid plaques and neurofibrillary tangles, were tested against wildtype controls for brain uptake of ${ }^{125}$ I-CQ BCA NPs. The in vivo uptake was imaged by autoradiography, and the data are given $\left(\mathrm{DLU} / \mathrm{mm}^{2}\right)$ in Table 4. Table 4 shows that at both 60 minutes and 90 minutes post injection of ${ }^{125} \mathrm{I}-\mathrm{CQ}$ BCA NPs, the AD transgenic mouse had a significantly greater brain uptake 
TABLE 4: 12 months triple transgenic Mice with ${ }^{125}$ ICQ BCA NPs in $\mathrm{DLU} / \mathrm{mm}^{2}($ mean $\pm \mathrm{SD}) ;(n=2)$.

\begin{tabular}{lcc}
\hline Time $(\mathrm{min})$ & Control & Triple transgenic \\
\hline 5 & $4.94 \times 10^{6} \pm 1.61 \times 10^{6}$ & $4.60 \times 10^{6} \pm 1.22 \times 10^{6}$ \\
30 & $1.53 \times 10^{5} \pm 8.26 \times 10^{3}$ & $1.77 \times 10^{5} \pm 1.33 \times 10^{4}$ \\
60 & $3.92 \times 10^{6} \pm 1.49 \times 10^{6}$ & $6.28 \times 10^{6} \pm 1.68 \times 10^{6}$ \\
90 & $3.94 \times 10^{6} \pm 5.05 \times 10^{5}$ & $4.85 \times 10^{6} \pm 1.00 \times 10^{5}$ \\
\hline
\end{tabular}

$P=.05 ; 1 \mathrm{mg} \mathrm{BCA} \mathrm{NP;} 2.24 \times 10^{6} \mathrm{CPM}^{125} \mathrm{ICQ}$.

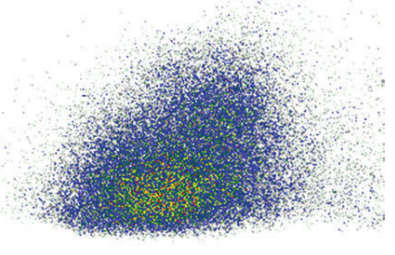

12 mo control

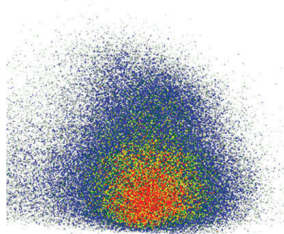

15 mo AD transgenic
FIGURE 5: ${ }^{125}$ ICQ BCA NPs (1 hour postinjection).

and retention of the imaging agent, as compared with the wildtype control. For example, at 60 minutes post injection, the brain uptake in the AD mouse was $6.28 \times 10^{6} \pm 1.68 \times 10^{6}$ versus $3.92 \times 10^{6} \pm 1.49 \times 10^{6}$ in the wildtype control.

4.3. In Vivo Imaging of the Amyloid Plaques in AD Mouse Models. The PBCA NPs were successfully loaded with the radiolabelled quinoline derivative ${ }^{125} \mathrm{I}-\mathrm{CQ}$ and delivered to the mice by intravenous administration. Storage Phosphor imaging qualitatively showed that the NPs transported the drug across the BBB. Further, the ${ }^{125} \mathrm{I}-\mathrm{CQ}$ labeled the amyloid deposits.

Figure 3 shows in vivo autoradiographs of the brain uptakes of ${ }^{125}$ ICQ BCA NPs and ${ }^{125}$ ICQ in transgenic mice (7 months old, APP/PS1), at 15 minutes postadministration of the nanoparticle-encapsulated drug and the free drug. The AD transgenic mice have a greater brain uptake with the use of nanoparticles, as compared to the free ${ }^{125} \mathrm{ICQ}$; thus, nanoparticulate encapsulation of ${ }^{125} \mathrm{ICQ}$ enhances $\mathrm{BBB}$ crossing of the drug. Furthermore, nanoparticulate encapsulation of ${ }^{125} \mathrm{ICQ}$ enhances retention of the drug. Figure 4 shows in vivo autoradiographs of the brain uptakes of ${ }^{125}$ ICQ BCA NPs and ${ }^{125}$ ICQ in transgenic mice (7 months old, $\left.\mathrm{APP} / \mathrm{PS} 1^{\star}\right)$, at 90 minutes post administration of the nanoparticle-encapsulated drug and the free drug. At 90 minutes post injection, the $\mathrm{AD}$ transgenic mice have a greater brain uptake with the nanoparticles, as compared to the free ${ }^{125} \mathrm{ICQ}$. Therefore, transgenic mice have longer brain retention of the nanoparticle delivered drug, as compared to the free drug. Figure 5 shows in vivo autoradiographs of the brain uptakes of ${ }^{125}$ ICQ BCA NPs in a 12-month wild type control mouse, and in a 15 -month $\mathrm{AD}$ transgenic mouse $\left(\mathrm{APP} / \mathrm{PS} 1^{\star}\right)$. At one hour post injection, the $\mathrm{AD}$ transgenic mouse has an increased brain uptake of the nanoparticles, presumably due to the presence of amyloid plaques.
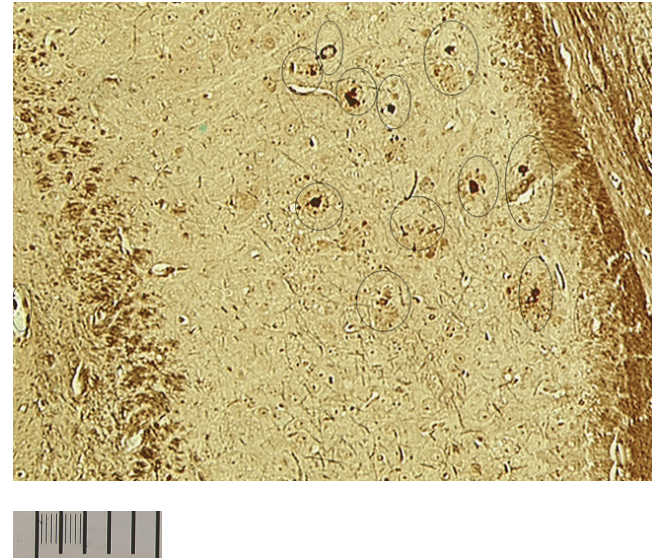

Figure 6: ${ }^{125}$ ICQ BCA NPs triple transgenic mouse model (mice aged 12 mo; 1 hour postinjection).

Figure 7: Hippocampal pathology in $3 \times$ Tg Bielschowsky Stain

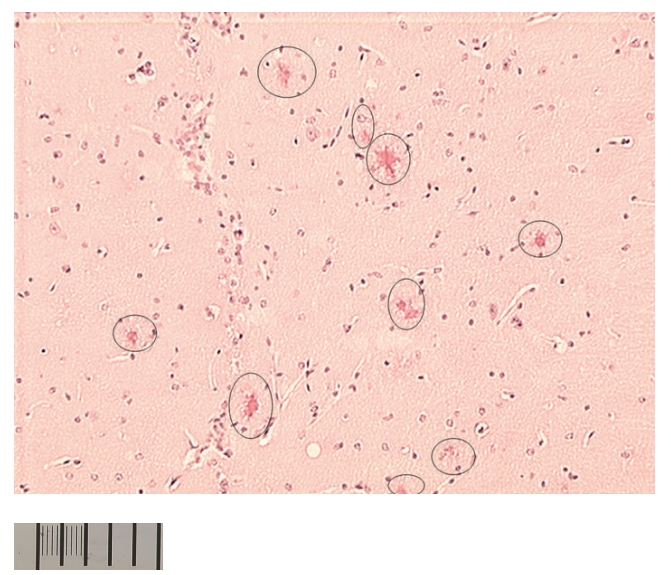

Figure 8: Aggregated amyloid plaques: 15 mo AD transgenic mouse. 


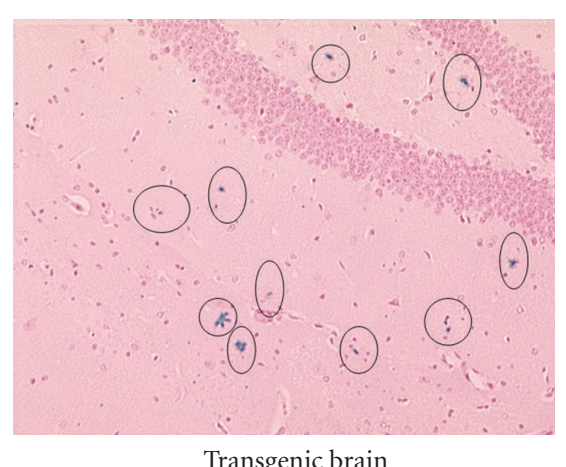

Transgenic brain

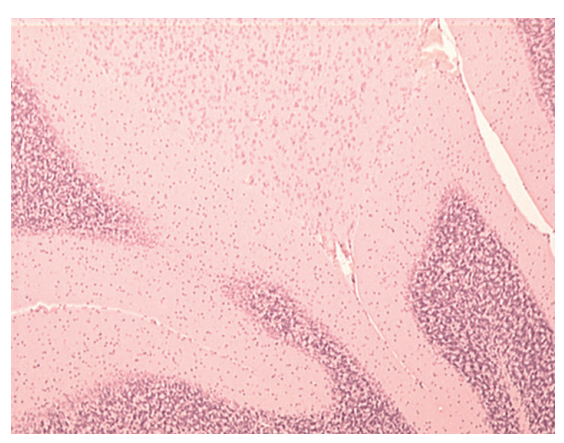

Control brain

\section{\|\|\|\|$\||| \mid 5 \mu \mathrm{m}$ slice at level of hippocampus}

FIgURE 9: 15 mo AD Transgenic: hippocampal staining of $\mathrm{Fe}^{2+}$.

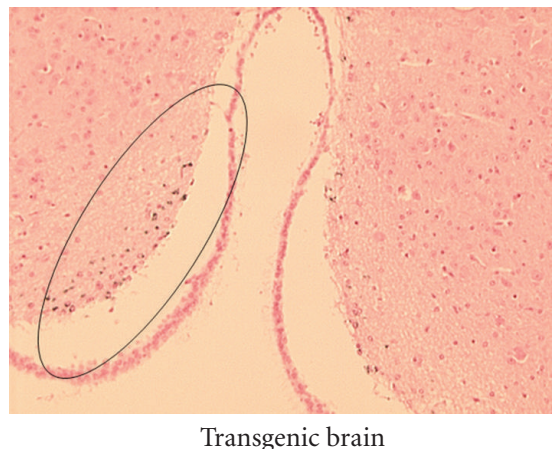

Transgenic brain

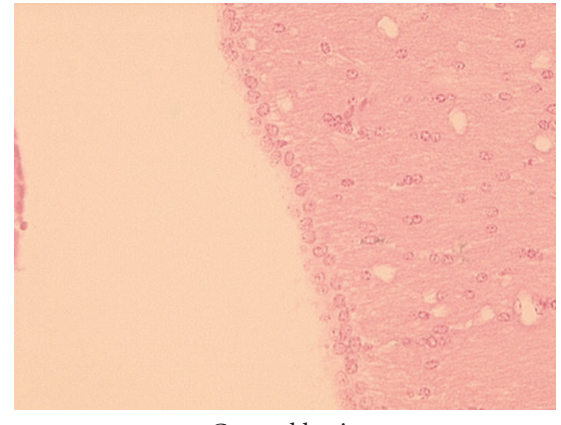

Control brain

$5 \mu \mathrm{m}$ slice at level of fourth ventricle

FIgURE 10: $\mathrm{Cu}^{2+}$ staining of aggregates in $3 \times \mathrm{Tg}(\mathrm{APP} / \mathrm{PS} 1 / \mathrm{Tau})$ mouse.

Experiments with ${ }^{125} \mathrm{ICQ}$ BCA NPs were also done in AD triple transgenic $(3 \times \mathrm{Tg})$ mice $(\mathrm{APP} / \mathrm{PS} 1 / \mathrm{Tau})$. In Figure 6, in vivo autoradiographs show that at one hour post injection, the $\mathrm{AD}$ triple Transgenic mouse had the higher brain uptake of the nanoparticles (as compared to the wild type control mouse). Additionally, a Bielchowsky stain (Figure 7) verified the $\mathrm{AD}$ pathology in the $3 \times \mathrm{Tg}$ mouse. Finally, histological staining of brain slices taken from these $\mathrm{AD}$ mouse models verified the presence of amyloid (Congo red, Figure 8), $\mathrm{Fe}^{2+}$ (Prussian blue, Figures 9 and 11), and $\mathrm{Cu}^{2+}$ (Rubeanic acid, Figure 10).

\section{Discussion}

Cyanoacrylate nanoparticles were specifically designed for carrying drugs across the blood-brain barrier in mouse models of Alzheimer's disease. In vivo biodistribution of the ${ }^{125} \mathrm{I}$-CQ-labelled butylcyanoacrylates in wild-type mice showed that they crossed the $\mathrm{BBB}$ with greater efficiency than the ${ }^{125}$ ICQ control. The BCA NP was selected as the prototype drug carrier because it polymerized with the most reproducibility; it crossed the BBB and had a rapid uptake and clearance from the normal brain. These parameters are important in order to validate the PBCA NP as a drug carrier across the BBB; another unique factor is the lipophilicity of the carrier. Good lipophilicity assists rapid uptake and clearance from the normal brain [17]. The lipophilicity of the nanoparticles was enhanced by surfactant coating with Tween-80.

The prototype PBCA NP was fully characterized for physicochemical and stabilizer effect. Smaller sized particles resulted when the monomer was polymerized at a lower $\mathrm{pH}$, in the presence of the stabilizer Dextran 70000 (as opposed to the hydrophilic polyethylene glycol, PEG), and definitely in the presence of a surfactant (e.g., Tween-80). Loading of the nanoparticles with amyloid-affinity drugs, such as derivatives of the Thioflavins (S- or T-), or Congo red did not significantly affect the size of the BCA NPs. Therefore, the BCA NPs maintained stability upon drug loading in vitro. Additional amyloid-affinity drugs have been reviewed in the literature $[2,17,18]$.

The amyloid-affinity chelator CQ was successfully radioiodinated with ${ }^{125} \mathrm{I}$; ${ }^{125} \mathrm{ICQ}$ was used in in vitro assays of human postmortem frontal cortex to test the affinity of the radiolabelled chelator for amyloid plaques. Autoradiography 


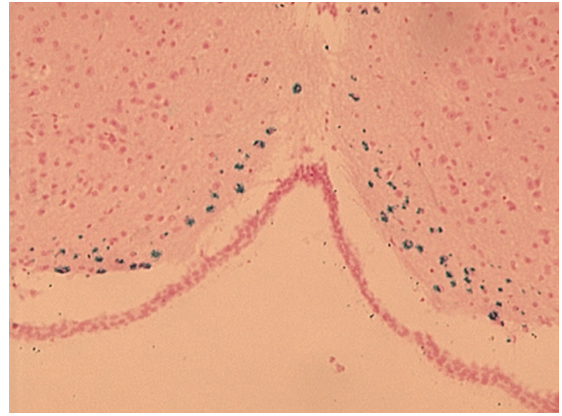

Transgenic brain

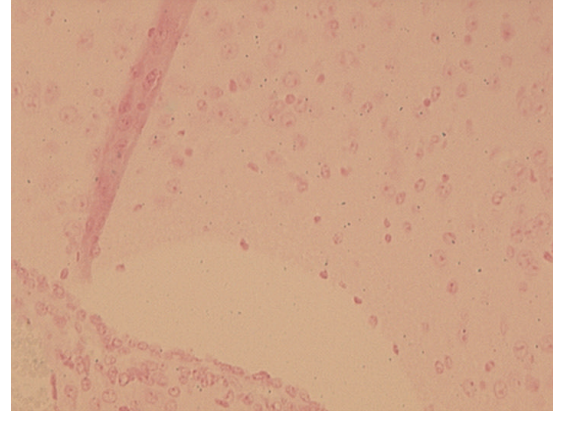

Control brain

$5 \mu \mathrm{m}$ slice at level of fourth ventricle

Figure 11: $\mathrm{Fe}^{2+}$ staining in $3 \times \mathrm{Tg}$ mouse.

validated preferential labeling of the $\mathrm{AD}$ tissue by the ${ }^{125}$ ICQ (compared with control brain tissue). Then, the ${ }^{125}$ ICQ was successfully encapsulated within PBCA NPs. ${ }^{125}$ ICQ PBCA NPs preferentially labeled frontal human AD tissue compared with frontal control tissue. PBCA NPs act as drug carriers of ${ }^{125} \mathrm{ICQ}$ targeted towards amyloid-beta plaques, presumably due to chelation of transitional metals in amyloid plaques.

\section{Conclusion}

In vivo detection of amyloid plaques for the early diagnosis of $\mathrm{AD}$ is desirable. Presently, histological confirmation of the plaques and of the neurofibrillary tangles is the only definitive mode of diagnosis. This is true despite the fact that patients typically receive clinical diagnoses based on cognitive tests, medical histories, and so forth. Noninvasive in vivo detection affords patients the opportunity to receive the most effective patient care as early as possible. Likewise, it allows clinicians the prospect of tracking disease progression when definitive treatment becomes available. Hence, healthcare professionals are able to design appropriate therapeutic strategies.

In vivo detection of amyloid-beta proteins improves specificity of diagnosis in noninvasive screening techniques, such as single photon emission computed tomography (SPECT) imaging. We have designed polybutylcyanoacrylate nanoparticles with incorporated radioligands and amyloid affinity agents that are attracted to the $\mathrm{A} \beta$ proteins. Thus, the clinical potential of the ${ }^{125} \mathrm{ICQ}-\mathrm{BCA}-\mathrm{NPs}$ is improved specificity of diagnostic accuracy in $\mathrm{AD}$ detection.

\section{Acknowledgments}

The authors would like to thank Tejraj Aminabhavi, Ph.D., David W. Russell, Ph.D., Charles White, M.D., Michael Bennett, M.D., Hilary Wilson, M.S., Perry N. Fuchs, Ph.D., Rebekkah Warren, B.S, John Shelton, B.S., and Thomas S. Harris, M.S.. This work was supported by NIH NIGMS 1 F31GM066381.

\section{References}

[1] R. L. Gutman, G. Peacock, and D. R. Lu, "Targeted drug delivery for brain cancer treatment," Journal of Controlled Release, vol. 65, no. 1-2, pp. 31-41, 2000.

[2] M. Ono, A. Wilson, J. Nobrega, et al., "11C-labeled stilbene derivatives as Abeta-aggregate-specific PET imaging agents for Alzheimer's disease," Nuclear Medicine and Biology, vol. 30, no. 6, pp. 565-571, 2003.

[3] Q. R. Smith, "A review of blood-brain barrier transport techniques," Methods in Molecular Medicine, vol. 89, pp. 193208, 2003.

[4] X. Huang, C. S. Atwood, M. A. Hartshorn, et al., "The A $\beta$ peptide of Alzheimer's disease directly produces hydrogen peroxide through metal ion reduction," Biochemistry, vol. 38, no. 24, pp. 7609-7616, 1999.

[5] C. W. Ritchie, A. I. Bush, A. Mackinnon, et al., "Metal-protein attenuation with iodochlorhydroxyquin (clioquinol) targeting $\mathrm{A} \beta$ amyloid deposition and toxicity in Alzheimer disease: a pilot phase 2 clinical trial," Archives of Neurology, vol. 60, no. 12, pp. 1685-1691, 2003.

[6] M. A. Lovell, J. D. Robertson, W. J. Teesdale, J. L. Campbell, and W. R. Markesbery, "Copper, iron and zinc in Alzheimer's disease senile plaques," Journal of the Neurological Sciences, vol. 158, no. 1, pp. 47-52, 1998.

[7] S. W. Suh, K. B. Jensen, M. S. Jensen, et al., "Histological evidence implicating zinc in Alzheimer's disease," Brain Research, vol. 852, pp. 274-278, 2000.

[8] R. A. Cherny, C. S. Atwood, M. E. Xilinas, et al., "Treatment with a copper-zinc chelator markedly and rapidly inhibits B-amyloid accumulation in Alzheimer's disease transgenic mice," Neuron, vol. 30, pp. 665-676, 2001.

[9] M. S. Yassin, J. Ekblom, M. Xilinas, C. G. Gottfries, and L. Oreland, "Changes in uptake of vitamin $\mathrm{B}_{12}$ and trace metals in brains of mice treated with clioquinol," Journal of the Neurological Sciences, vol. 173, no. 1, pp. 40-44, 2000.

[10] A. I. Bush, "Metal complexing agents as therapies for Alzheimer's disease," Neurobiology of Aging, vol. 23, no. 6, pp. 1031-1038, 2002.

[11] A. Kontush, C. Berndt, W. Weber, et al., "Amyloid- $\beta$ is an antioxidant for lipoproteins in cerebrospinal fluid and plasma," Free Radical Biology and Medicine, vol. 30, no. 1, pp. 119-128, 2001. 
[12] C. Opazo, S. Luza, V. L. Villemagne, et al., "Radioiodinated clioquinol as a biomarker for $\beta$-amyloid: $\mathrm{Zn}^{2+}$ complexes in Alzheimer's disease," Aging Cell, vol. 5, no. 1, pp. 69-79, 2006.

[13] J. Kreuter, R. Alyautdin, D. Kharkevich, et al., "Passage of peptides through the blood brain barrier with collodial polymer particles (nanoparticles)," Brain Research, vol. 674, pp. 171-174, 1995.

[14] W. E. Klunk, B. J. Lopresti, M. D. Ikonomovic, et al., "Binding of the positron emission tomography tracer Pittsburgh compound- $B$ reflects the amount of amyloid- $\beta$ in Alzheimer's disease brain but not in transgenic mouse brain," Journal of Neuroscience, vol. 25, no. 46, pp. 10598-10606, 2005.

[15] J. Ahi, J. Radulovic, and J. Spiess, "The role of hippocampal signaling cascades in consolidation of fear memory," Behavioural Brain Research, vol. 149, no. 1, pp. 17-31, 2004.

[16] T. Blank, I. Nijholt, K. Eckart, and J. Spiess, "Priming of long-term potentiation in mouse hippocampus by corticotropin-releasing factor and acute stress: implications for hippocampus-dependent learning," Journal of Neuroscience, vol. 22, no. 9, pp. 3788-3794, 2002.

[17] G. Henriksen, B. H. Yousefi, A. Drzezga, and H.-J. Wester, "Development and evaluation of compounds for imaging of $\beta$ amyloid plaque by means of positron emission tomography," European Journal of Nuclear Medicine and Molecular Imaging, vol. 35, supplement 1, pp. S75-S81, 2008.

[18] A. Nordberg, "PET imaging of amyloid in Alzheimer's disease," Lancet Neurology, vol. 3, no. 9, pp. 519-527, 2004. 


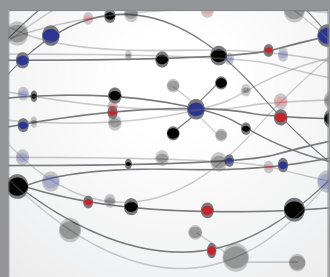

The Scientific World Journal
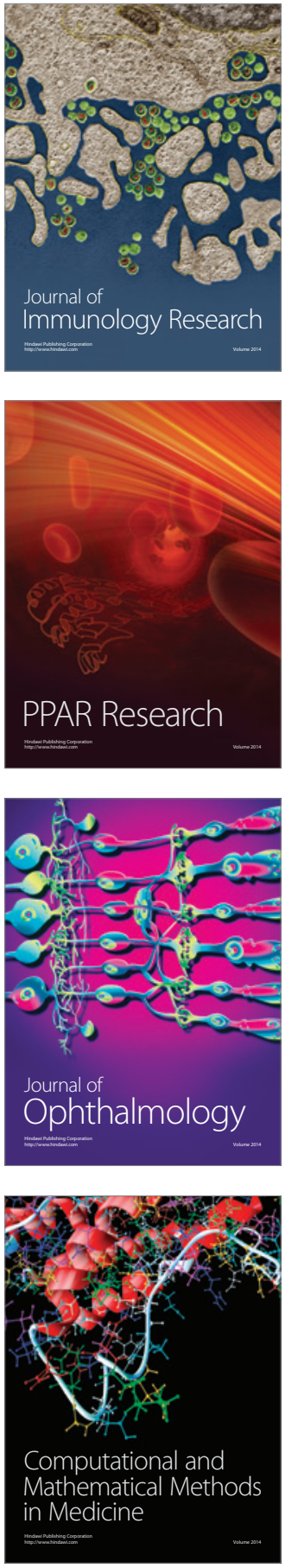

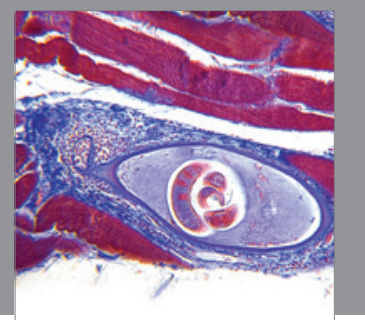

Gastroenterology

Research and Practice
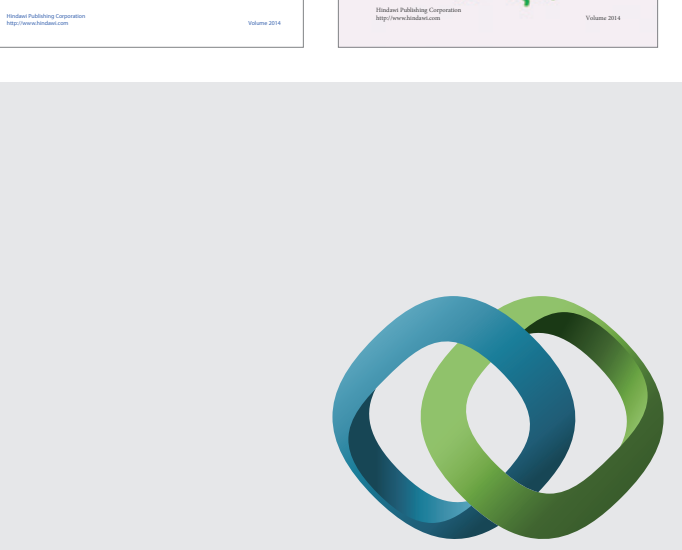

\section{Hindawi}

Submit your manuscripts at

http://www.hindawi.com
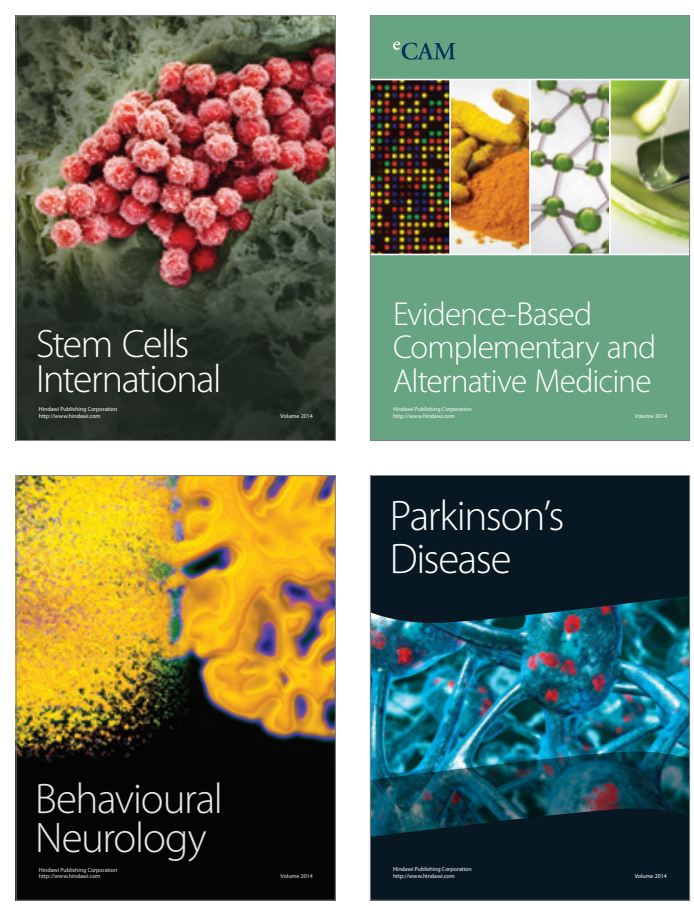

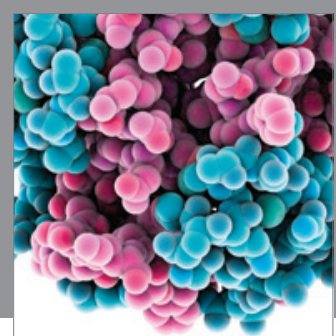

Journal of
Diabetes Research

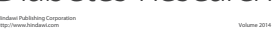

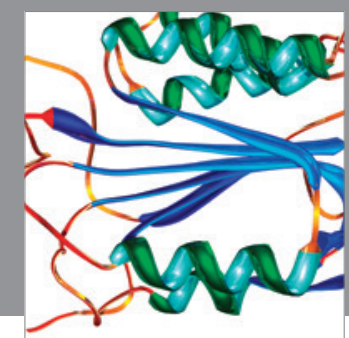

Disease Markers
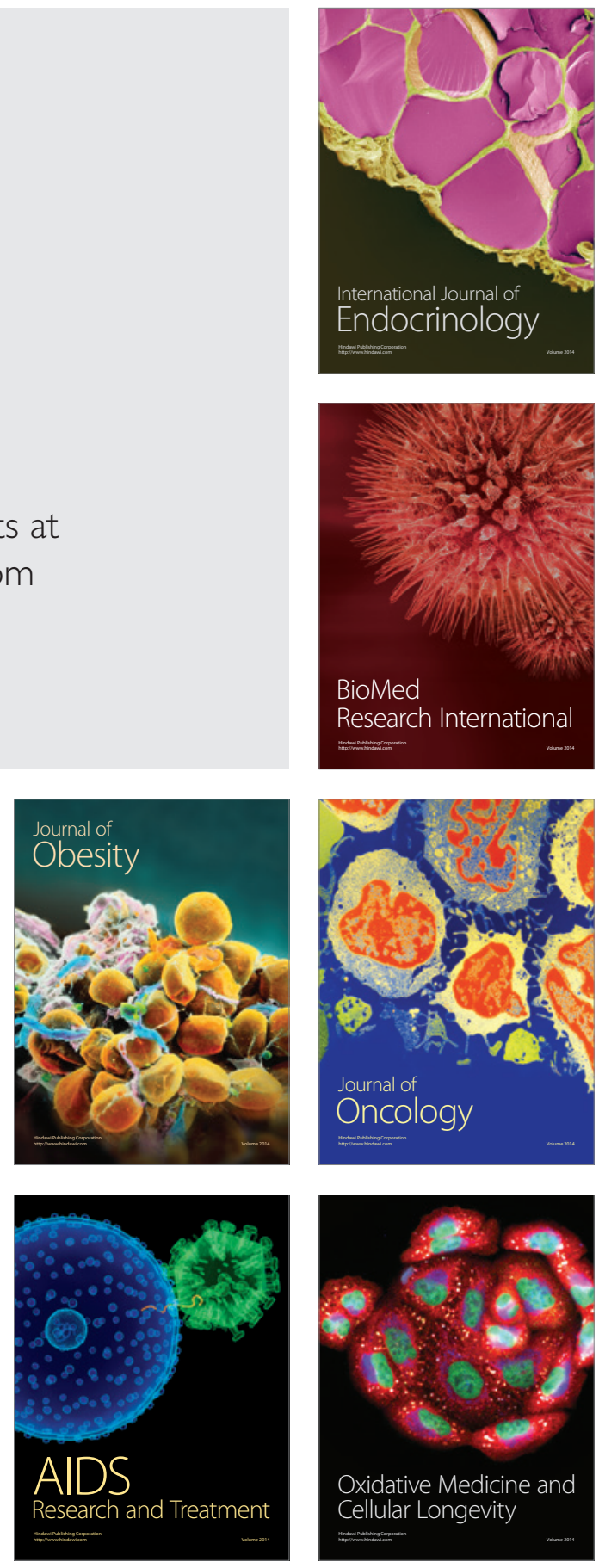